\title{
A FURTHER RESULT ON EXTENDING EXPANSIVE HOMEOMORPHISMS
}

\author{
J. D. WINE
}

\begin{abstract}
In this note we give a necessary and sufficient condition for a selfhomeomorphism defined on an open subset of a compact metric space to be extended to the whole space using the concept of a generator. Two examples of homeomorphic extensions of expansive homeomorphisms are given, one examining the equivalence of the theorem and the other showing that a previous result's sufficient condition for the extension of expansive homeomorphisms is not necessary.
\end{abstract}

1. Introduction. Since 1962 there have been works by Bryant [2], Williams [7], and Wine [10] wherein the extension of expansive homeomorphism was investigated. The result of this note gives a necessary and sufficient condition for the extension of an expansive homeomorphism using the concept of a generator introduced by Keynes and Robertson [3].

Definition 1. A homeomorphism $h$ of a metric space $(X, \rho)$ onto itself is expansive with expansive constant $\delta$ if given any two distinct points $x$ and $y$ of $X$ there is an integer $n$ such that $\rho\left(h^{n}(x), h^{n}(y)\right)>\delta$.

Definition 2. Let $h$ be a homeomorphism of the compact metric space $(X, \rho)$ onto itself. A finite open cover $\mathscr{U}$ of $X$ is a generator for $(X, h)$ if for each bisequence $\left\{A_{i}\right\}$ of elements of $\mathscr{U}$, the intersection $\bigcap_{i=-\infty}^{\infty} h^{-i}\left(\overline{A_{i}}\right)$ is at most one point.

Definition 3. If $h$ is a homeomorphism of the metric space $(X, \rho)$ onto itself and $x$ is a point of $X$, then the orbit of $x$ is $\bigcup\left\{h^{n}(x) \mid n\right.$ is an integer $\}$. Notation is $O(x)$.

DEFINITION 4. For a homeomorphism of the metric space $(X, \rho)$ onto itself, the set $\left\{x_{\alpha} \in X \mid \alpha \in A\right\}$ is an orbital basis of $(X, \rho)$ with respect to $h$ if $\bigcup\left\{O\left(x_{\alpha}\right) \mid \alpha \in\right.$ $A\}=X$ and $\alpha$ not equal to $\beta$ implies $O\left(x_{\alpha}\right)$ not equal to $O\left(x_{\beta}\right)$.

The following theorem, characterizing expansiveness is found in [ 3 and 4 ].

THEOREM 1. Let $h$ be a homeomorphism of the compact metric space $(X, \rho)$ onto itself. Then $h$ is expansive if and only if there is a generator for $(X, h)$.

2. Result. We now use Theorem 1 to prove the following result concerning the extension of expansive homeomorphisms.

THEOREM 2. Let $(X, d)$ be an open subspace of the compact metric space $(Y, \rho)$, let $h$ be an expansive homeomorphism of $(X, d)$ onto itself with expansive constant $\delta$, and let

Received by the editors August 28, 1984 and, in revised form, November 12, 1984.

1980 Mathematics Subject Classification. Primary 54C10; Secondary 54H20.

Key words and phrases. Expansive homeomorphisms, extending homeomorphisms, generator. 
$\hat{h}$ be a homeomorphic extension of $h$ to $Y$ such that $\hat{h}$ restricted to $Z=Y-X$ is expansive with expansive constant $\delta$. Then $\hat{h}$ will be expansive on $Y$ with expansive constant $\delta$ if and only if every generator of $(Z, \hat{h})$ which has the form $\mathscr{U}=\left\{A_{\alpha} \cap Z \mid A_{\alpha}\right.$ is open in $\left.Y, \operatorname{diam}\left(A_{\alpha}\right)<\delta, \alpha \in \mathscr{A}\right\}$ has the property that, for every bisequence of elements of $\mathscr{U},\left\{A_{i} \cap Z\right\}_{i=-\infty}^{\infty}, \cap_{i=-\infty}^{\infty} \hat{h}^{-i}\left(\overline{A_{i}}\right)$ is at most one point.

Proof. Necessity. Suppose $\hat{h}$ is expansive on $Y$, and that $\mathscr{U}$ has the form given in the statement of the theorem. Extend $\left\{A_{\alpha} \mid A_{\alpha}\right.$ is used in $\left.\mathscr{U}\right\}$ to an open cover $\mathscr{G}$ of $Y$, where

$$
\mathscr{G}=\left\{G_{\beta} \mid \operatorname{diam}\left(G_{\beta}\right)<\delta, \beta \in \mathscr{B}\right\}
$$

and $G_{\beta}$ not in $\mathscr{U}$ implies $G_{\beta}$ is disjoint from $Z$. Let $\mathscr{G}^{*}$ be a finite subcover of $\mathscr{G}$. Return to $\mathscr{G}^{*}$ any of the $A_{\alpha}$ in $\mathscr{U}$ that may have been removed from $\mathscr{G}$ by the taking of the finite subcover, and call the new cover $\mathscr{H}$. The cover $\mathscr{H}$ is finite with all elements having diameter less than $\delta$. Since $\hat{h}$ is expansive on $Y$ with expansive constant $\delta$, the cover $\mathscr{H}$ is a generator for $(Y, \hat{h})$. Therefore, $\mathscr{H}$ has the property that for any bisequence $\left\{A_{i}\right\}_{i=-\infty}^{\infty}$ of elements of $\mathscr{H}$ the intersection $\bigcap_{i=-\infty}^{\infty} \hat{h}^{-i}\left(\overline{A_{i}}\right)$ consists of at most one point. Since $\mathscr{U}$ is a subset of $\mathscr{H}$, it must be the case that $\mathscr{U}$ has the desired property also.

Sufficiency. Let $\mathscr{G}^{*}$ be an open cover of $Y$ by the open balls of radius $\delta / 2$ and let $\mathscr{G}$ be a finite subcover of $\mathscr{G}^{*}$. Let $\mathscr{U}$ be the subset of $\mathscr{G}$ which consists of all elements of $\mathscr{G}$ which have nonvoid intersection with $Z=Y-X$. Then the family $\hat{\mathscr{U}}=\left\{G_{\lambda} \cap\right.$ $Z \mid G_{\lambda}$ is in $\left.\mathscr{U}\right\}$ is a generator for $\hat{h}$ restricted to $Z$, since $\hat{h}$ is expansive on $Z$ with expansive constant $\delta$.

Now let $S=\left\{A_{k}\right\}_{k=-\infty}^{\infty}$ be any bisequence from $\mathscr{G}$ and suppose that the intersection $\cap\left\{\hat{h}^{-k}\left(\overline{A_{k}}\right) \mid A_{k}\right.$ is in $\left.S\right\}$ contains distinct points $x$ and $y$. By hypothesis, if $S$ is a subset of $\mathscr{U}$, the intersection would contain at most one point. Therefore, it must be the case that at least one of the $A_{k}$ in $S$ must not be in $\mathscr{U}$. Hence, the intersection must be contained in $X$. However, $h$ is expansive on $X$ with expansive constant $\delta$, so there exists an integer $n$ such that $d\left(h^{n}(x), h^{n}(y)\right)>\delta$. This implies that $\hat{h}^{n}(x)$ and $\hat{h}^{n}(y)$ cannot both be elements of the set $\bar{A}_{n}$. A contradiction has been reached, and it must be that the intersection can contain at most one point. Hence $\mathscr{G}$ is a generator for $\hat{h}$ on $Y$, and $\hat{h}$ is expansive on $Y$ with expansive constant $\delta$.

3. Examples. In [10] the following theorem is proved. We give an example which shows that the condition given in this theorem is not necessary.

THEOREM 3. Let $(X, \rho)$ be a subspace of the metric space $(Y, d)$ and let $h$ be an expansive homeomorphism of $(X, \rho)$ onto itself with expansive constant $\delta$. Suppose $\hat{h}$ is a homeomorphic extension of $h$ to $(Y, d)$. Then $f$ is expansive with expansive constant $\delta$ if

(1) $f$ restricted to $Y-X$ is expansive with expansive constant $\delta$, and

(2) there is an orbital basis $B$ of $X$ with respect to $h$ such that $d(x, Y-X)>\delta$ for every $x$ in $B$. 
ExAmple 1. Let $S=\{u \mid u=1-1 / k, k=2,3,4, \ldots\} \cup\{u \mid u=1 / k, k=$ $2,3,4, \ldots\}$, let $X=S \times S$, and let $(X, \rho)$ be $X$ considered as a subspace of $R^{2}$. If $p$ is in $S$, let $p^{\prime}$ be the least element of $S$ greater than $p$. Define the homeomorphism $h$ taking $(X, \rho)$ onto itself by $h(p, q)=\left(p^{\prime}, q^{\prime}\right)$ for every point $(p, q)$ in $X$. The homeomorphism $h$ is expansive on $(X, \rho)$ with expansive constant $\delta$ for any $\delta$ less than $1 / 6$.

Let $(Y, d)$ be the closure of $(X, \rho)$ considered as a subspace of $R^{2}$, and let $\hat{h}$ be the extension of $h$ to $(Y, d)$ defined by

$$
\begin{array}{rlrl}
\hat{h}(x, 0) & =(x, 0), & x=0,1, \\
\hat{h}(x, 1) & =(x, 1), \quad x=0,1, \\
\hat{h}(x, p) & =\left(x, p^{\prime}\right), \quad x=0,1 \text { and } p \text { in } S, \\
\hat{h}(p, y) & =\left(p^{\prime}, y\right), \quad y=0,1 \text { and } p \text { in } S .
\end{array}
$$

The extension $\hat{h}$ is a homeomorphism on $(Y, d)$, and $\hat{h}$ is expansive on $Y-X$ with the expansive constant $\delta$ for $\delta$ less than $1 / 6$. It is also the case that $h$ is expansive on $(Y, d)$ for the same $\delta$ 's, however, the orbit $O(1 / k, 1-1 / k)$, where $1 / k<\delta$, is always within $\delta$ of $Y-X$.

EXAMPLE 2. We finally consider a simple example in which all the hypotheses of Theorem 2 are satisfied except the equivalence condition on the generators.

For $n$ a positive integer less than or equal to nineteen, define $C_{n}$ to be a subset of $R^{2}$ by

$$
C_{n}=\left\{(r, \theta) \mid r=\frac{1}{n}, \theta=0, \pi, \text { and } \pm \frac{k \pi}{2(k+1)}, k=1,2, \ldots, n\right\},
$$

and define

$$
C=\left\{(r, \theta) \mid r=\frac{1}{20}, \theta=0, \pi, \text { and } \pm \frac{k \pi}{2(k+1)}, k=1,2, \ldots, 19\right\} .
$$

Let $Y=\left\{\bigcup_{n=1}^{19} C_{n}\right\} \cup C$ and obtain the space $(Y, \rho)$ as a subspace of $R^{2}$.

Let the subspace $(X, d)$ be given by the union of the $C_{n}, X=\cup_{n=1}^{19} C_{n}$. Define the homeomorphism $h$ taking $(X, d)$ onto itself by $h(p)=h(1 / n, \theta)=\left(1 / n, \theta^{\prime}\right)$, where $\left(1 / n, \theta^{\prime}\right)$ is the next point counterclockwise from $(1 / n, \theta)$. Then $h$ is expansive with expansive constant $\delta=\sqrt{2} / 20$.

The extension $\hat{h}$ of $h$ defined on $Z=Y-X$ by $\hat{h}(1 / 20, \theta)=\left(1 / 20, \theta^{\prime}\right)$ is homeomorphic and expansive on $Z$ with expansive constant $\delta=\sqrt{2} / 20$, but $h$ is not expansive on $Y$ for that expansive constant.

Let $A_{\alpha}=\{(1 / 19, \alpha),(1 / 20, \alpha)\}$ for each $\alpha$ an angle for $C_{19}$. Then $\operatorname{diam}\left(A_{\alpha}\right)<\delta$ and $\mathscr{U}=\left\{A_{\alpha} \cap Z \mid \alpha\right.$ an angle for $\left.C_{19}\right\}$ is a generator for $\hat{h}$ on $Z$. Now define the bisequence $\left\{B_{i}\right\}_{i=-\infty}^{+\infty}$ of elements of $\left\{A_{\alpha} \mid \alpha\right.$ is an angle for $\left.C_{19}\right\}$ by $B_{0}=A_{0}$, $B_{i} \cap Z=\hat{h}^{i}\left(B_{0} \cap Z\right), i$ an integer. Then

$$
\bigcap_{i=-\infty}^{\infty} \hat{h}^{-i}\left(\bar{B}_{i}\right)=\left\{\left(\frac{1}{20}, 0\right),\left(\frac{1}{19}, 0\right)\right\} .
$$




\section{REFERENCES}

1. B. F. Bryant and P. Walters, Asymptotic properties of expansive homeomorphisms, Math. Systems Theory 3 (1969), 60-66.

2. B. F. Bryant, Expansive self-homeomorphism of a compact metric space, Amer. Math. Monthly 69 (1962), 386-391.

3. H. B. Keynes and J. B. Robertson, Generators for topological entropy and expansiveness, Math. Systems Theory 3 (1969), 51-59.

4. W. L. Reddy, Lifting expansive homeomorphisms to symbolic flows, Math. Systems Theory 2 (1968), 91-92.

5. W. R. Utz, Unstable homeomorphisms, Proc. Amer. Math. Soc. 1 (1950), 769-774.

6. Expansive mappings, Topology Proc. 3 (1978), 221-226.

7. R. K. Williams, On expansive homeomorphisms, Amer. Math. Monthly 76 (1969), 176-178.

8. Some results on expansive mappings, Proc. Amer. Math. Soc. 26 (1970), 655-663.

9. ___ Further results on expansive mappings, Proc. Amer. Math. Soc. 56 (1976), 284-288.

10. J. D. Wine, Extending expansive homeomorphisms, Proc. Amer. Math. Soc. 86 (1982), 531-534.

Department of Mathematics, University of Wisconsin - LaCrosse, LaCrosse, Wisconsin 54601 